Supporting Information for

\title{
Physiologically Relevant Mechanics of Biodegradable Polyester Nanoparticles
}

Nourin Alsharif, ${ }^{\dagger}$ Behnaz Eshaghi, ${ }^{*}$ Björn M. Reinhard, ${ }^{*}$ and Keith A. Brown ${ }^{* \$}$

${ }^{\dagger}$ Department of Mechanical Engineering, Boston University, Boston, Massachusetts 02215, United States

${ }^{\dagger}$ Department of Chemistry and the Photonics Center, Boston University, Boston, Massachusetts, 02215, United States

${ }^{\S}$ Physics Department and Division of Materials Science and Engineering, Boston University, Boston, Massachusetts 02215, United States

\section{$\underline{\text { Table of Contents }}$}

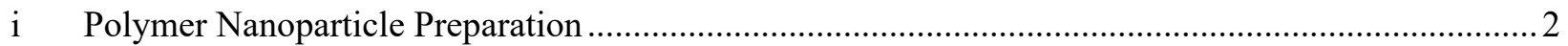

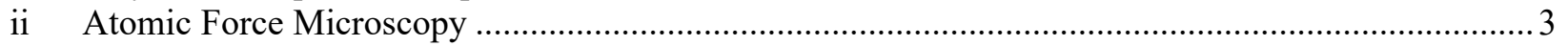

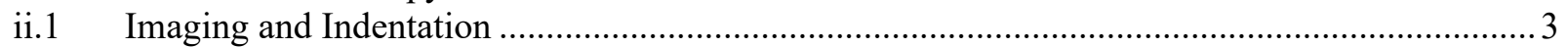

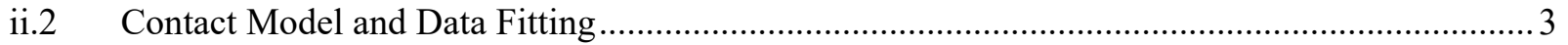

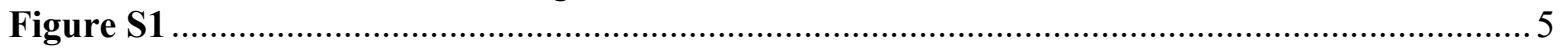

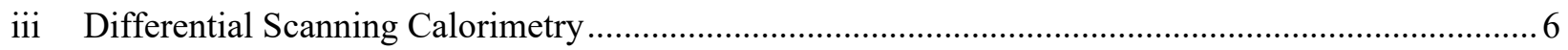

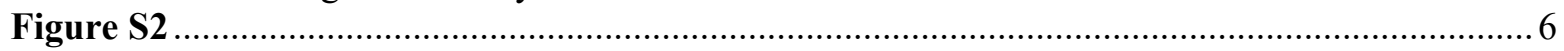

iv $\quad$ Literature Reports of Modulus vs. Temperature (Figure S3) _......................................................... 7

v Modulus Range Determination for Elevated Temperature Experiments in Air.................................. 8

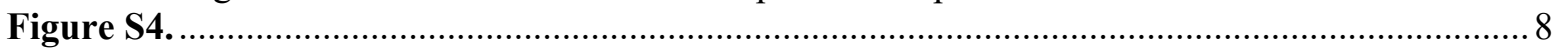

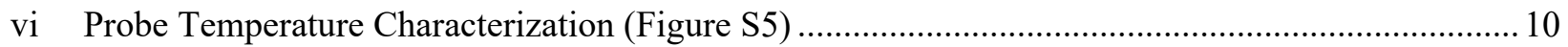

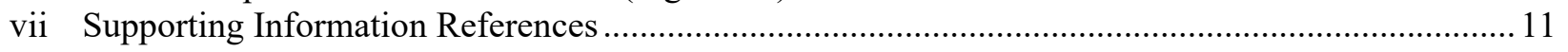




\section{i Polymer Nanoparticle Preparation}

Polymer nanoparticles (NPs) were prepared following previously published procedures. ${ }^{1,2}$ In short, $0.4 \mathrm{~mL}$ of a $2.5 \mathrm{mg} / \mathrm{mL}$ polymer solution in acetonitrile was added dropwise to water. The final solution was sonicated in a bath sonicator for $5 \mathrm{~min}$. Next, NPs were washed 3 times (4000 g - $15 \mathrm{~min})$ using an Amicon Ultra-4 centrifugal filter (MilliporeSigma, Burlington, MA) with a molecular weight cutoff of $10 \mathrm{kDa}$ to concentrate the NP solution. The polymers used in this work include PLGA (poly (D,L-lactide-coglycolide) lactide:glycolide 50:50, ester terminated, RG503, with intrinsic viscosity of $0.32-0.44 \mathrm{dL} / \mathrm{g}$ and MW of 24,000-38,000 Da), PLA (poly(D,L-lactide), ester terminated, R203S with intrinsic viscosity of $0.25-0.35 \mathrm{dL} / \mathrm{g}$ and molecular weight of 18,000-28,000 Da) and high molecular weight PLA (poly(D,Llactide) ester terminated, R207S with intrinsic viscosity of 1.3-1.7 dL/g and molecular weight of $209,000 \mathrm{Da}^{3}$ ). All polymers were purchased from Sigma-Aldrich (St Louis, MO). 


\section{ii Atomic Force Microscopy}

\section{ii.1 Imaging and Indentation}

Samples were prepared by 20 min incubation of polymer NPs on $0.1 \%$ poly-L-lysine (Sigma-Aldrich, St Louis, MO) treated circular cover slips (Asylum Research). Following the incubation, unbound NPs were removed by successive washes. Concentration of NPs used for AFM studies were determined by iterative experiments of incubation and imaging to identify concentrations that would yield a sizeable number of particles on the surface without overlap or aggregation. NPs used for hydrated indentation experiments were kept hydrated in Milli-Q water throughout the duration of preparation and experimentation, while dried NPs were dried after washing. Surfaces were first placed on a Peltier heater attachment (Asylum Research, CoolerHeater) integrated with an AFM (Oxford Instruments - MFP3D Infinity AFM) and left to equilibrate with the AFM head in place for $20 \mathrm{~min}$. After this time, a tapping mode height scan of the surface was conducted to identify regions without NPs where the probe could be calibrated using quintuplicate indentations on the rigid cover slip (for optical lever sensitivity) and a measurement of the thermal power spectral density. For measurements on softer polymers (hydrated PLGA and PLA $A_{\text {LMw }}$ at $37^{\circ} \mathrm{C}$ ), probes with stiffness $k=0.08 \mathrm{~N} / \mathrm{m}$ (Asylum Research, BL-AC40TS) were used, for stiff polymers in water, probes with $k=0.9 \mathrm{~N} / \mathrm{m}$ (MikroMasch, CSC37/Al BS) were used, and for all polymers in air, probes with $k=15 \mathrm{~N} / \mathrm{m}$ (MikroMasch, NSC35 Hard/Al) were used.

After calibration, an area scan of $2 \times 2 \mu \mathrm{m}^{2}$ was performed at $1 \mathrm{~Hz}$ in tapping mode to locate a sizeable number of particles (Figure 1a). Next, the tallest NP in the scan was identified using AFM software and a high resolution scan of the particle was conducted (Figure 1b). The diameter of the AFM tip was calculated by subtracting the height of the NP $d$ from its measured width $w$ (both measured using a line scan from the height channel). Calculated AFM tip radii ranged from 20 to $40 \mathrm{~nm}$ based on duration of use. Once the survey scan had been completed, a magnified area of $250 \times 250 \mathrm{~nm}^{2}$ (or a size encompassing the particle) was scanned at $6 \mathrm{~Hz}$ in tapping mode to image a single NP. The scan rate was increased to reduce the frequency of contact between the tip and NP to prevent spatial manipulation. The highest point of the NP was once again found using AFM software, and subsequent force curves with a force set point of 5-10 $\mathrm{nN}$ at $0.04 \mathrm{~Hz}$, depending on the precieved stiffness of the particle, were carried out at the point of maximum height of the NP. The NP was scanned immediately after indentation to check for movement or drift. If the particle was observed to have moved appreciably as a result of drift or as a result of the measurement, the indentation data for that NP was not used. The probe was then moved to another location on the surface and the imaging and indentation process was repeated. If the tip radius was calculated to be larger than 50 $\mathrm{nm}$, the probe was replaced.

\section{ii.2 Contact Model and Data Fitting}

Data in the form of force and indentation was exported from the AFM software. The force and indentation were calculated using the calibration of the optical lever sensitivity and stiffness conducted on every probe prior to use. ${ }^{4}$ To isolate ${ }^{5}$ the modulus of the NP, the following equation ${ }^{6}$ was used to calculate the NP modulus:

$$
F=E_{N P} \times\left[\frac{4(h * b)^{\frac{3}{2}} R_{t, N P}^{\frac{1}{2}}}{3\left(1-v_{N P}^{2}\right)}\right]
$$


where $F$ is the force exerted by the AFM probe, $E_{N P}$ is the modulus of the NP, $h$ is the indentation depth measured by the AFM, and $v_{\mathrm{NP}}$ is the Poisson's ratio of the NP. Poisson's ratio for PLGA ${ }^{7}$ and $\mathrm{PLA}^{8}$ were taken to be 0.25 and 0.35 , respectively. The term $b$ accounts for the curvature of the indenter and NP and is given by:

$$
b=\frac{R_{N P}^{\frac{1}{3}}}{R_{t, N P}^{\frac{1}{3}}+R_{N P}^{\frac{1}{3}}}
$$

where $R_{N P}$ is the radius of the NP, calculated from the height of the NP under test, and $R_{t, N P}$ is calculated as:

$$
R_{t, N P}=\frac{R_{t} R_{N P}}{R_{t}+R_{N P}}
$$

where $R_{t}$ is the radius of the tip. Here, $R_{t}$ is calculated as the difference between the NP's measured width and its height as the measured width of the NP is represents a convolution of the shapes of the tip and the NP (Figure 1d). The force-indentation curve was fit from its initial contact point, $h=0$ to $R_{N P} / 20$, to avoid substrate effects. ${ }^{9}$

As there are no comparable measurements of polyester NPs in physiological conditions, it is important to verify the model and ensure that the resultant $E$ is accurate. To do so, we compare the resultant modulus from the modified double contact model (equation S1) to a Hertzian contact model between two spheres (assuming the AFM probe tip is spherical) as follows:

$$
F=\frac{\frac{4}{3} E R_{e f f}^{\frac{1}{2}} h^{\frac{3}{2}}}{1-v^{2}}
$$

Where $R_{\text {eff }}$ is the effective radius between the two spheres as follows:

$$
\frac{1}{R_{e f f}}=\frac{1}{R_{N P}}+\frac{1}{R_{t}}
$$

In addition to comparisons with the original Hertzian contact model between a sphere and half space as follows:

$$
F=\frac{\frac{4}{3} E R_{t}^{\frac{1}{2}} h^{\frac{3}{2}}}{1-v^{2}}
$$

We fit the data from indentation experiments on dried PLA $A_{L M W}$ NPs at $25^{\circ} \mathrm{C}$ to all three models (equations S1, S4, and S6). Importantly, without accounting for the two interfaces of the NP (NP and AFM tip, NP and substrate), the models over predict the modulus in comparison to previously reported values of PLA ${ }^{10,11}$ (Figure S1) as previously discussed. ${ }^{6}$ 


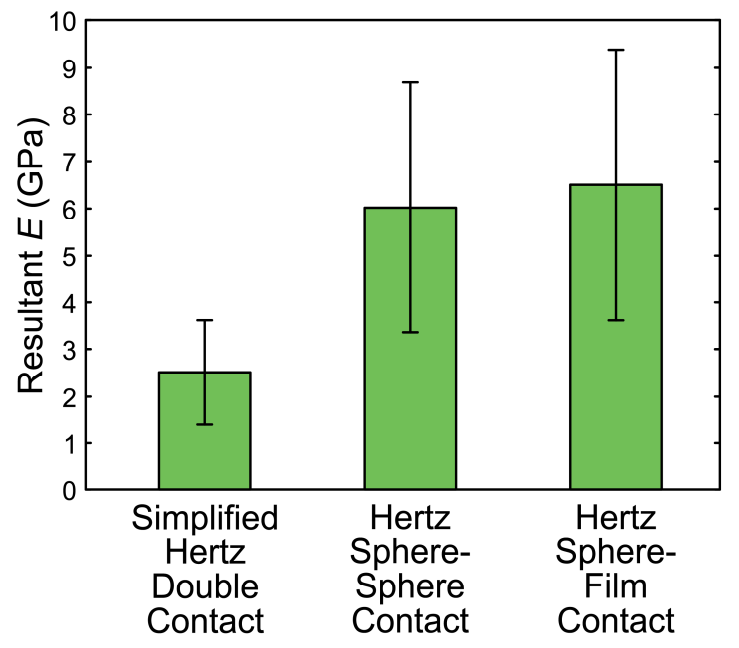

Figure S1. Comparison of various contact models, shown for the model polymer PLA $A_{L M w}$ at $25^{\circ} \mathrm{C}$ in dried conditions. Green bars represent resultant $E$ found for PLA $\mathrm{LMW}_{\mathrm{LM}} \mathrm{NPs}$ at $25^{\circ} \mathrm{C}$ modeled with the Hertz double contact model (equation S1), Hertz sphere-sphere contact model (equation S4), and the Hertz sphere-plane model (equation S6). 


\section{iii Differential Scanning Calorimetry}

A thorough description of the differential scanning calorimetry (DSC) characterization processes has been previously discussed. ${ }^{2}$ In short, to characterize thermal behavior of polymer NPs, the routine NP synthesis was concentrated by a factor of 3 . For DSC measurements of NPs in solution, $30 \mu \mathrm{L}$ of solution was transferred into an aluminum pan (Thermal Support, Hayesville, NC) and sealed. For the measurements on dried NPs, $30 \mu \mathrm{L}$ of the NPs solution was transferred to the DSC pan and air dried for $4 \mathrm{~h}$, following $4 \mathrm{~h}$ further drying at $35{ }^{\circ} \mathrm{C}$ before sealing the pans. For polymer powder measurements, samples were sealed in the pans. Thermograms were recorded from $25^{\circ} \mathrm{C}$ to $75{ }^{\circ} \mathrm{C}$, at a heating rate of $5{ }^{\circ} \mathrm{C} / \mathrm{min}$ by using a Mettler Toledo Polymer DSC R (Mettler-Toledo, Columbus, OH). DSC software was used to determine the glass transition temperatures. Determination of glass transition temperature $T_{\mathrm{g}}$ at the minima of the DSC thermogram peak (Figure S2).

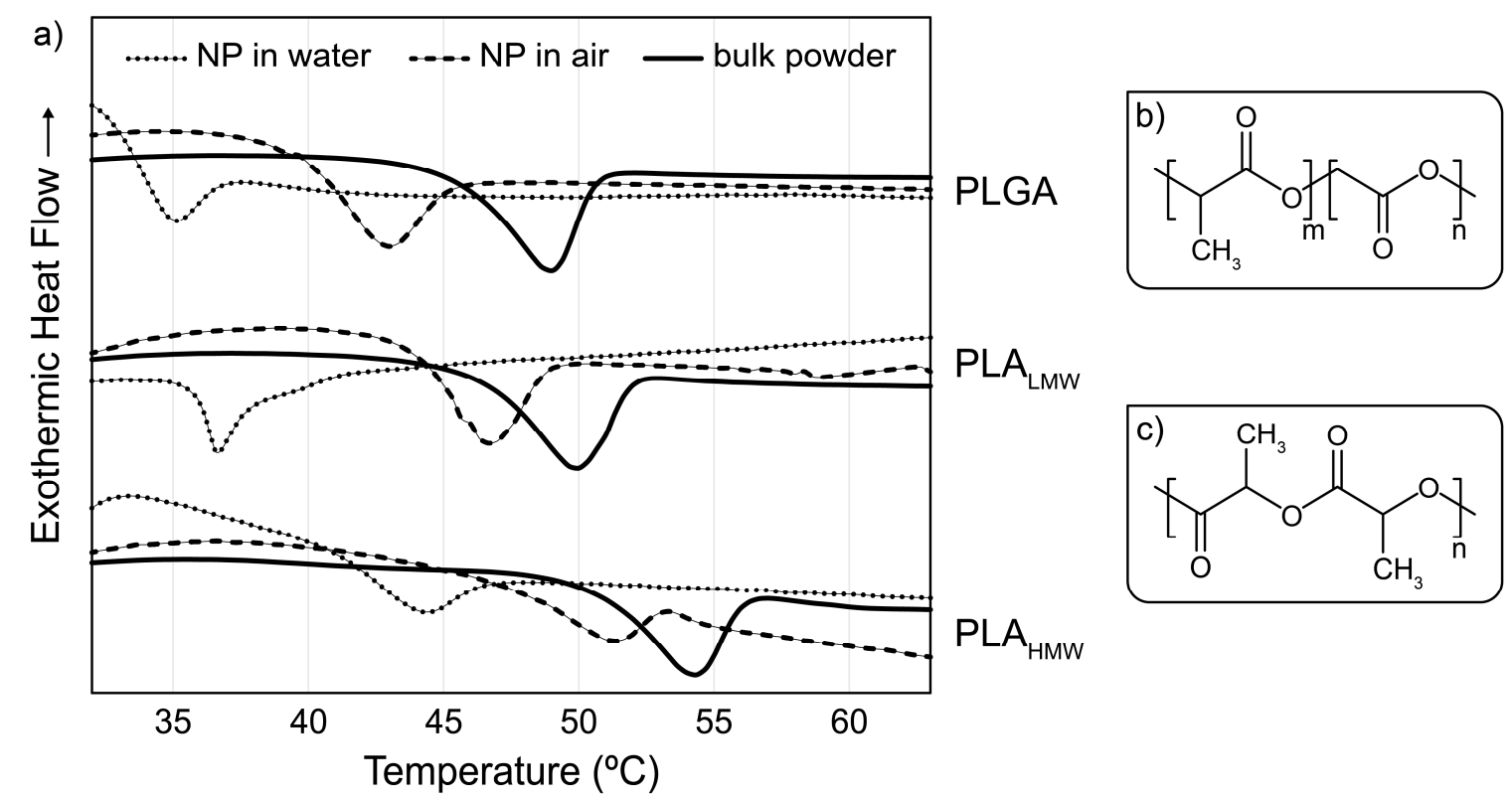

Figure S2. a) Differential scanning calorimetry (DSC) thermograms for 3 types of polymer nanoparticles (NPs) vs. temperature. Dashed lines represent hydrated NPs, dotted represents dried NPs, and solid lines represent bulk powder. In all cases, the glass transition temperature $T_{\mathrm{g}}$ is measured at the minima of the peaks. Molecular structure for b) poly(lactide-co-glycolide), PLGA NPs and c) poly(lactic acid), PLA NPs. 


\section{iv Literature Reports of Modulus vs. Temperature (Figure S3)}
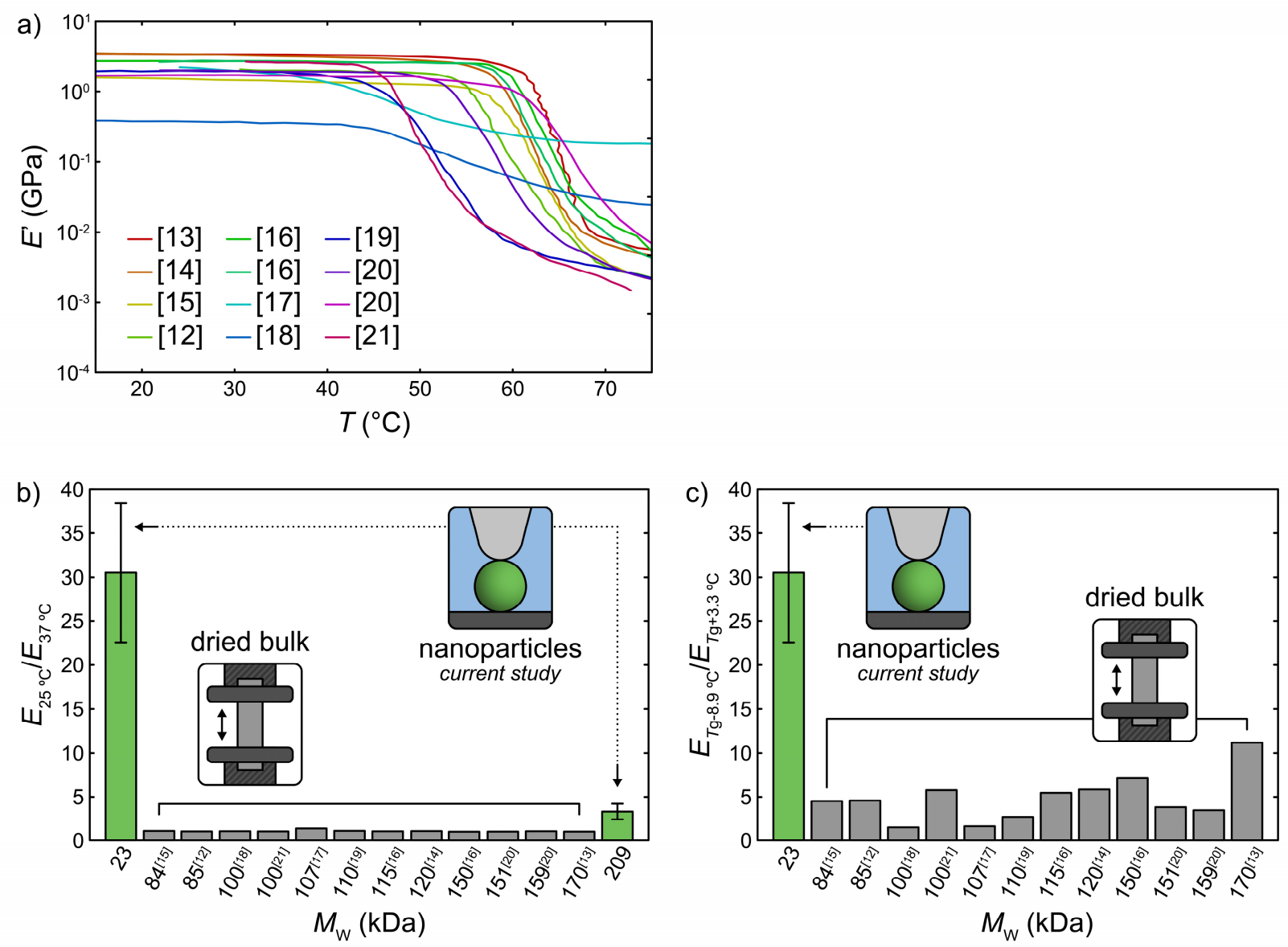

Figure S3. a) Digitized storage modulus $E^{\prime}$ from previous reports ${ }^{12-21}$ vs. temperature $T$. Ratio of $E$ at 25 and $37^{\circ} \mathrm{C}$ from (a) used for $E_{25^{\circ} \mathrm{C}} / E_{37^{\circ} \mathrm{C}}$ in b) across different molecular weights ( $\left.M_{\mathrm{W}}\right)$ of PLA. Data from nanoparticle measurements from this work (PLALMw NPs in water at $37^{\circ} \mathrm{C}$ and in air at $25{ }^{\circ} \mathrm{C}$ ) are also presented for reference in green. c) Comparison of $E^{\prime}$ above $\left(37^{\circ} \mathrm{C}-T_{\text {g,PLA NPs }}=3.3{ }^{\circ} \mathrm{C}\right)$ and below $\left(T_{\text {g,PLA }}\right.$ NPs $\left.-25{ }^{\circ} \mathrm{C}=8.9{ }^{\circ} \mathrm{C}\right) T_{\mathrm{g}}$ of each respective polymer from a) using the onset method. Data from NP measurements from this work is also presented for reference in green. All legend entries correspond to references in Supporting Information (and included in main text references). 
Indentation experiments at elevated temperatures in air, as described in Figure 4, yield force-indentation behavior not readily modeled by existing contact models. This difficulty is due to the hypothesized heterogeneity of temperature and subsequently, mechanics, of the nanoparticle, rendering its elastic response significantly more complex than those accurately described as Hookean contacts. A sample forcedistance curve for a PLA $\mathrm{LMW}_{\mathrm{LP}} \mathrm{NP}$ in air at a stage temperature set point of $49.9^{\circ} \mathrm{C}$ in Figure $\mathrm{S} 4$ exhibits long range adhesion.

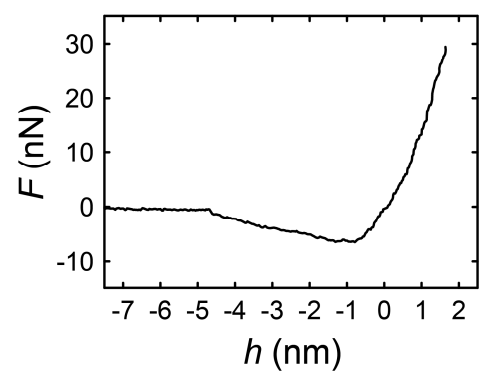

Figure S4. Typical force $F$ vs. indentation $h$ curve for $\mathrm{PLA}_{\mathrm{LMW}} \mathrm{NPs}$ at $49.9^{\circ} \mathrm{C}$ in air.

To estimate $E$ of the NPs at elevated temperatures in air, we use a bounding technique to provide a range of moduli values that could feasibly describe the NP E. It is important to note that there are assumptions of material uniformity within these models that are not met in practice due to the hypothesized heterogeneity of NP heating. To establish an upper bound of $E$, we assume that the NP follows a Hertzian contact model with $E$ at the substrate interface equal to $E$ of the NP at the AFM tip interface. Accordingly, a modified JKR model was used to account for the adhesion observed in the Force $F$-indentation $h$ curves (Figure S4): ${ }^{6}$

$$
h=\left(\frac{F}{K R_{12}^{\frac{1}{2}}}\right)^{\frac{2}{3}}+\left(\frac{1}{K R_{32}^{\frac{1}{2}}}\left(F+4 F_{a d}+\sqrt{8 F_{a d} F+\left(4 F_{a d}\right)^{2}}\right)\right)^{\frac{2}{3}}-\left(\frac{12 \gamma \pi}{K}\right)^{\frac{2}{3}} R_{32}^{\frac{1}{3}}
$$

where

$$
K=\frac{4 E}{3\left(1-v^{2}\right)}
$$

and adhesive force $F_{\text {ad }}$ defined as:

$$
F_{a d}=\frac{3}{2} \pi \gamma R_{32}
$$


where $\gamma$ is the work of adhesion. For a more nuanced perspective of the indentation process, we can also assume that $E$ at the AFM tip interface corresponds to a cooler material than that at the substrate interface. Therefore, we assume that $E$ describing the AFM tip interface (the first iteration of $K$ in eqn. S7) is fixed at a value commensurate with the measurement of $E$ of the NP at $37{ }^{\circ} \mathrm{C}$ in air. Therefore, the fitting of the force-indentation curves yield a modulus value describing only the JKR interface between the NP and the substrate, which we have chosen to present as the lower bound of our range in Figure 4a. Importantly, the large range represented by the upper and lower bounds presented here are indicative of the uncertainties associated with fitting the $F$ - $h$ response of a thermally and elastically heterogonous NP with models that cannot account for a thermal and modulus gradient. 


\section{vi Probe Temperature Characterization (Figure S5)}
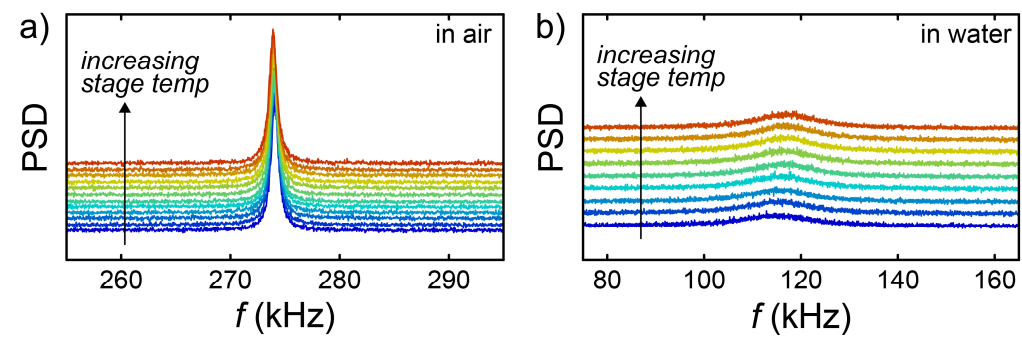

Figure S5. Power spectral densities for a probe $40 \mu \mathrm{m}$ away from heated stage a) in air and b) in water. Red shift in color distribution represents increasing stage temperature, the precise values of which are denoted in Figure $4 \mathrm{~b}$ in the main text. Fitting to these spectra enabled calculation of probe temperature. 
(1) Fang, R. H.; Aryal, S.; Hu, C.-M. J.; Zhang, L. Quick Synthesis of Lipid-Polymer Hybrid Nanoparticles with Low Polydispersity Using a Single-Step Sonication Method. Langmuir 2010, 26 (22), 16958-16962.

(2) Eshaghi, B.; Alsharif, N.; An, X.; Akiyama, H.; Brown, K. A.; Gummuluru, S.; Reinhard, B. M. Stiffness of HIV-1 Mimicking Polymer Nanoparticles Modulates Ganglioside-Mediated Cellular Uptake and Trafficking. Adv. Sci. 2020, 7 (18), 2000649.

(3) Bragagni, M.; Beneitez, C.; Martín, C.; de la Ossa, D. H. P.; Mura, P. A.; Gil-Alegre, M. E. Selection of PLA Polymers for the Development of Injectable Prilocaine Controlled Release Microparticles: Usefulness of Thermal Analysis. Int. J. Pharm. 2013, 441 (1-2), 468-475.

(4) Alsharif, N.; Burkatovsky, A.; Lissandrello, C.; Jones, K. M.; White, A. E.; Brown, K. A. Design and Realization of 3D Printed AFM Probes. Small 2018, 14 (19), 1-6.

(5) Dokukin, M. E.; Guz, N. V.; Sokolov, I. Quantitative Study of the Elastic Modulus of Loosely Attached Cells in AFM Indentation Experiments. Biophys. J. 2013, 104 (10), 2123-2131.

(6) Glaubitz, M.; Medvedev, N.; Pussak, D.; Hartmann, L.; Schmidt, S.; Helm, C. A.; Delcea, M. A Novel Contact Model for AFM Indentation Experiments on Soft Spherical Cell-like Particles. Soft Matter 2014, 10 (35), 6732-6741.

(7) Brady, M. A.; Renzing, A.; Douglas, T. E. L.; Liu, Q.; Wille, S.; Parizek, M.; Bacakova, L.; Kromka, A.; Jarosova, M.; Godier, G.; Warnke, P. H. Development of Composite Poly(LactideCo-Glycolide)-Nanodiamond Scaffolds for Bone Cell Growth. J. Nanosci. Nanotechnol. 2015, 15 (2), 1060-1069.

(8) Pillin, I.; Montrelay, N.; Bourmaud, A.; Grohens, Y. Effect of Thermo-Mechanical Cycles on the Physico-Chemical Properties of Poly(Lactic Acid). Polym. Degrad. Stab. 2008, 93 (2), 321-328.

(9) Li, L.; Encarnacao, L. M.; Brown, K. A. Polymer Nanomechanics: Separating the Size Effect from the Substrate Effect in Nanoindentation. Appl. Phys. Lett. 2017, 110 (4), 043105.

(10) Farah, S.; Anderson, D. G.; Langer, R. Physical and Mechanical Properties of PLA, and Their Functions in Widespread Applications - A Comprehensive Review. Adv. Drug Deliv. Rev. 2016, 107, 367-392.

(11) Van de Velde, K.; Kiekens, P. Biopolymers: Overview of Several Properties and Consequences on Their Applications. Polym. Test. 2002, 21 (4), 433-442.

(12) Li, Y.; Xin, S.; Bian, Y.; Dong, Q.; Han, C.; Xu, K.; Dong, L. Stereocomplex Crystallite Network in Poly(D,L-Lactide): Formation, Structure and the Effect on Shape Memory Behavior and Enzymatic Hydrolysis of Poly(D,L-Lactide). RSC Adv. 2015, 5 (31), 24352-24362.

(13) Li, Y.; Shimizu, H. Improvement in Toughness of Poly(1-Lactide) (PLLA) through Reactive Blending with Acrylonitrile-Butadiene-Styrene Copolymer (ABS): Morphology and Properties. Eur. Polym. J. 2009, 45 (3), 738-746.

(14) Suryanegara, L.; Nakagaito, A. N.; Yano, H. The Effect of Crystallization of PLA on the Thermal and Mechanical Properties of Microfibrillated Cellulose-Reinforced PLA Composites. Compos. Sci. Technol. 2009, 69 (7-8), 1187-1192.

(15) Mofokeng, J. P.; Luyt, A. S. Dynamic Mechanical Properties of PLA/PHBV, PLA/PCL, PHBV/PCL Blends and Their Nanocomposites with TiO2 as Nanofiller. Thermochim. Acta 2015, 613, 41-53.

(16) Blaker, J. J.; Lee, K.-Y.; Walters, M.; Drouet, M.; Bismarck, A. Aligned Unidirectional PLA/Bacterial Cellulose Nanocomposite Fibre Reinforced PDLLA Composites. React. Funct. Polym. 2014, 85, 185-192.

(17) Albano, C.; González, G.; Palacios, J.; Karam, A.; Covis, M. PLLA-HA vs. PLGA-HA Characterization and Comparative Analysis. Polym. Compos. 2013, 34 (9), 1433-1442.

(18) Nakafuku, C.; Takehisa, S. Y. Glass Transition and Mechanical Properties of PLLA and PDLLAPGA Copolymer Blends. J. Appl. Polym. Sci. 2004, 93 (5), 2164-2173. 
(19) Du, K.; Gan, Z. Shape Memory Behaviour of HA-g-PDLLA Nanocomposites Prepared via in Situ Polymerization. J. Mater. Chem. B 2014, 2 (21), 3340-3348.

(20) Urayama, H.; Kanamori, T.; Kimura, Y. Properties and Biodegradability of Polymer Blends of Poly(L-Lactide)s with Different Optical Purity of the Lactate Units. Macromol. Mater. Eng. 2002, 287 (2), 116-121.

(21) Liu, Y.; Li, Y.; Chen, H.; Yang, G.; Zheng, X.; Zhou, S. Water-Induced Shape-Memory Poly(d,1Lactide)/Microcrystalline Cellulose Composites. Carbohydr. Polym. 2014, 104 (1), 101-108. 